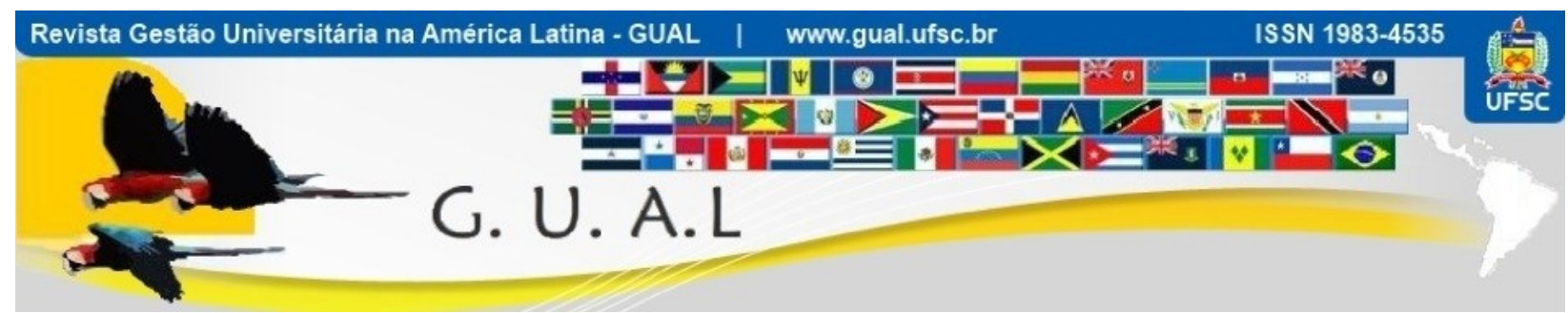

DOI: http://dx.doi.org/10.5007/1983-4535.2018v11n1p48

\title{
O PAPEL DAS IES NO DESENVOLVIMENTO SUSTENTÁVEL: ESTUDO DE CASO DA UNIVERSIDADE FEDERAL DE SANTA MARIA
}

\author{
THE ROLE OF HEI IN SUSTAINABLE DEVELOPMENT:CASE STUDY OF \\ FEDERAL UNIVERSITY OF SANTA MARIA
}

Fernando Gazzoni, Mestre Universidade Federal de Santa Maria - UFSM gazzoni.adm@hotmail.com

Flavia Luciane Scherer, Doutora Universidade Federal de Santa Maria - UFSM flaviascherer@globo.com

Ivanete Schneider Hahn, Doutora Universidade do Alto Vale do Rio do Peixe - UNIARP ivischneider@hotmail.com

Aletéia de Moura Carpes, Doutora Centro Universitário Franciscano - UNIFRA alecarpes.adm@hotmail.com

Maríndia Brachak dos Santos, Mestra Universidade Federal de Santa Maria - UFSM marindiabrachak@gmail.com

Recebido em 24/fevereiro/2016

Aprovado em 07/novembro/2017

Sistema de Avaliação: Double Blind Review

Esta obra está sob uma Licença Creative Commons Atribuição-Uso. 


\title{
RESUMO
}

As Instituições de Ensino Superior (IES) possuem papel fundamental na formação de pensamentos e opiniões, sendo um dos principais órgãos que podem potencializar o desenvolvimento de um pensamento sustentável. Neste sentido, este estudo buscou investigar o grau de conhecimento que os servidores da Universidade Federal de Santa Maria (UFSM) apresentam sobre temáticas sustentáveis da Administração Pública. Investigou-se ainda, como as práticas de desenvolvimento sustentável propostas para as IES são adotadas pelos servidores da UFSM. A operacionalização da pesquisa ocorreu por meio de um estudo de caso único e holístico, utilizando como unidade de análise a UFSM. A coleta de dados envolveu levantamentos por meio de documentos da IES, arquivos e questionário aplicado junto a 649 servidores da UFSM. Os resultados evidenciam que há ampla deficiência no conhecimento referente a temáticas sustentáveis da Administração Pública. Complementarmente, verificouse que grande parte dos respondentes não desenvolvem atividades rotineiras visando a racionalização dos recursos utilizados, sendo que foram identificadas algumas iniciativas individuais, mas que não necessariamente refletem no grupo. Neste caso, pode-se concluir que a busca pelo desenvolvimento sustentável na UFSM caminha a passos lentos.

Palavras-chave: Sustentabilidade. Gestão de Ies. Conhecimento.

\begin{abstract}
Higher Education Institutions (HEI) play a fundamental role in the formation of thoughts and opinions, being one of the main organs that can enhance the development of sustainable thinking. Thus, this study aimed to investigate the degree of knowledge that the servers of the Federal University of Santa Maria (UFSM) present on sustainable thematic Public Administration. It investigated yet, as the practices of sustainable development proposals for HEI are adopted by the UFSM servers. The operationalization of the research was through a study of unique and holistic case, using as unit of analysis the UFSM. Data collection involved surveys through documents of HEI files and questionnaire addressed to 649 servers of UFSM. The results show that there is wide deficiency of knowledge regarding sustainable thematic Public Administration. In addition, it was found that most respondents do not develop routine activities aimed at rationalization of the resources used, and identified some individual initiatives, but do not necessarily reflect the group. In this case, it can be concluded that the pursuit of sustainable development in UFSM walks at a slow pace.
\end{abstract}

Keywords: Sustainability. Management of Hei. Knowledge. 


\section{INTRODUÇÃO}

A busca desenfreada pelo desenvolvimento econômico nos últimos anos degradou e poluiu o meio ambiente. Ocorreu uma utilização abusiva dos recursos naturais, de forma descontrolada, e essa situação trouxe diversas consequências, que podem ser visualizadas através dos problemas ambientais atuais (SANTOS, 2007). Em decorrência destes problemas, gestão ambiental e desenvolvimento sustentável passaram a serem elementos de atenção por parte de gestores e pesquisadores, mesmo que muitas vezes motivados por interesses diversos ou obrigados por força legal ou social (SILVA et al., 2011). Adicionalmente, a sustentabilidade de ações e programas desenvolvidos pela Administração Pública, passou a ser uma das principais preocupações dos cidadãos (COUTO; COELHO, 2015).

A Administração Pública é responsável pela utilização de grande parte dos recursos disponíveis para o desenvolvimento das suas atividades. A implantação de programas socioambientais nos órgãos públicos é categórica para o desenvolvimento do país, pois tais medidas refletirão no orçamento da União, visto que transformações provindas da esfera comportamental evitarão desperdícios, reduzindo assim diferentes custos (CAVALCANTI, 2012).

Neste processo, as Instituições de Ensino Superior (IES) possuem papel fundamental, por serem formadoras de pensamento e opinião, sendo assim, podem potencializar a criação e a difusão de um pensamento sustentável. Para tanto, é necessário que as pessoas envolvidas no desenvolvimento das atividades universitárias sirvam como base para a disseminação do conhecimento e fortalecimento de práticas sustentáveis. Embora tenha havido alguns desenvolvimentos na educação superior no que concerne ao desenvolvimento sustentável, ainda existem numerosos desafios que precisam ser superados (LEAL FILHO; MANOLAS; PACE, 2015).

Enquanto base deste processo, as IES devem ser reconhecidas por sua busca ao desenvolvimento sustentável e consequentemente, devem colocar em prática suas crenças em relação à sustentabilidade e servir de base, isto é, exemplo aos discentes e a sociedade. Neste sentido, as IES têm um papel preponderante no desenvolvimento sustentável e devem ser, elas próprias, modelos de sustentabilidade para a sociedade (FOUTO, 2002).

Para que as IES incorporem os preceitos do desenvolvimento sustentável é necessário que estes conceitos sejam empregados nas atividades rotineiras desenvolvidas pelos servidores nas suas unidades de trabalho. Atitudes como reaproveitamento de material, 
economia de água e energia elétrica são atitudes simples e que podem ser desenvolvidas na unidade onde o servidor desenvolve o seu trabalho. A adoção de critérios ambientais nas atividades administrativas e operacionais da Administração Pública constitui-se um processo de melhoramento contínuo que consiste em adequar os efeitos ambientais das condutas do poder público à política de prevenção de impactos negativos ao meio ambiente (MMA, 2009, p. 26).

Outra prática que se faz necessária para a busca do desenvolvimento sustentável é a disseminação do conhecimento referente às temáticas sustentáveis, principalmente aquelas ligadas à Administração Pública. A percepção de desenvolvimento sustentável é formada pelo pensamento, valores e atitudes e são modificados com base no conhecimento adquirido, para então resultar em ações práticas. (DOBES, 2001). Além de serem difundidos entre os discentes, aspectos sustentáveis devem ser compartilhados entre os servidores da instituição, é necessário que se coloque em prática e se desenvolva conceitos abordados no ensino. Desta maneira é que será possível o desenvolvimento de uma conscientização ambiental (GALLI, 2008), haja vista que a conscientização ambiental, decorrente das organizações e da sociedade, é a chave para o desenvolvimento sustentável e a própria sustentabilidade.

Em decorrência desta situação, este estudo tem como objetivo investigar o grau de conhecimento que os servidores da Universidade Federal de Santa Maria (UFSM) apresentam sobre as temáticas sustentáveis da Administração Pública. Buscou-se investigar ainda, como as práticas de desenvolvimento sustentável propostas para as IES são adotadas e implementadas pelos servidores da UFSM em seus ambientes de trabalho.

Ao focar essa temática o artigo proporciona uma discussão sobre o tema e assim soma esforços à literatura de desenvolvimento sustentável. Contribui ainda para o desenvolvimento de uma gestão pautada na racionalização de recursos e na conscientização ambiental das pessoas envolvidas nos processos de trabalho das IES.

\section{DESENVOLVIMENTO SUSTENTÁVEL}

Um dos conceitos mais utilizados para definição de desenvolvimento sustentável, tido como a origem do termo, é o apresentado no Relatório de Brundtland (CMMAD, 1991, p. 46): “desenvolvimento sustentável é o desenvolvimento que satisfaz as necessidades da geração presente sem comprometer a capacidade das gerações futuras para satisfazer as suas 
próprias necessidades". Neste relatório foram definidas ainda as ações e metas a serem desenvolvidas pelos Estados em âmbito mundial (JACOBI, 2003).

Um dos conceitos mais utilizados, quando se trata das dimensões da sustentabilidade é o proposto por Sachs (1993), que defende um novo paradigma para o planejamento do desenvolvimento baseado em cinco dimensões e que devem ser consideradas simultaneamente. De modo resumido essas dimensões são:

- Social: tem como objetivo de melhorar substancialmente os direitos e condições das amplas massas de população e reduzir as distâncias entre os padrões de vida de abastados e não abastados;

- Econômica: possibilitada por uma alocação e gestão eficiente dos recursos e por um fluxo regular do investimento público e privado, avaliada mais em termos macrossociais, do que apenas por critérios de lucratividade microempresarial;

- Ecológica: envolvendo medidas para reduzir o consumo de recursos e a produção de resíduos, medidas para intensificar as pesquisas e a introdução de novas tecnologias limpas e poupadoras de recursos;

- Espacial: voltada a uma configuração rural-urbana mais equilibrada e uma melhor distribuição territorial de assentamentos humanos e atividades econômicas, envolvendo questões de concentração excessiva das áreas metropolitanas, destruição de ecossistemas frágeis, projetos de agricultura, industrialização descentralizada e rede de proteção das reservas naturais, biosfera e biodiversidade;

- Cultural: em busca das raízes endógenas dos modelos de modernização e dos sistemas rurais integrados de produção, privilegiando processos de desenvolvimento que respeitem as peculiaridades de cada ecossistema, de cada cultura e cada local.

De comum a todos os conceitos propostos pelos autores, têm-se as variáveis econômica, social e ambiental. Estas variáveis formam o triple bottom line, conceito proposto por Elkington (1999). Para o autor, essas variáveis são os três pilares norteadores de decisões e ações relacionadas à gestão organizacional, aproximando o conceito de responsabilidade social corporativa do conceito da sustentabilidade. Conforme Dias (2008), o mais importante, na abordagem das três dimensões da sustentabilidade empresarial, é o equilíbrio dinâmico entre as dimensões econômica, social e ambiental.

Nesse sentido, há uma diversidade de entendimentos no que se refere às principais dimensões inseridas da sustentabilidade. Na literatura, verificam-se diversos modelos, sendo 
que Azevedo (2002) propôs as dimensões econômica, social, ambiental, ética, cultural, temporal e espacial. Deponti e Almeida (2002) reduziram as dimensões para: econômica, social, cultural e ambiental. Consalter (2008) reuniu as variáveis social e cultural em uma única variável, definindo as dimensões em sociocultural, técnico agronômica, ecológica e econômica.

Verifica-se que o desenvolvimento sustentável não se trata de uma escolha entre a proteção ambiental e o progresso social, mas de um esforço maior para desenvolvimento econômico e social que seja compatível com a proteção ambiental (CIEGIS; RAMANAUSKIENE; MARTINKUS, 2009).

\subsection{AS IES E O DESENVOLVIMENTO SUSTENTÁVEL}

As IES são organismos de grande importância no desenvolvimento da sociedade, como um dos principais agentes transformadores de profissionais que ditarão os rumos do mercado e da sociedade. Elas têm o papel de qualificar e conscientizar os cidadãos que serão os futuros formadores de opinião (TAUCHEN; BRANDLI, 2006).

Não obstante, as IES são entendidas como formadoras de opinião e meio de contribuição para qualificação do pensamento dos seus egressos, bem como, encontram-se envolvidas com o desenvolvimento sustentável (TAUCHEN; BRANDLI, 2006). Ao assumir sua "característica organizacional as IES, compreendem que a sustentabilidade socioambiental faz-se necessária, uma vez que essas instituições são responsáveis pela formação cidadã, política, cultural e social de seus egressos" (FREITAS, 2013, p. 21).

Dentro de uma visão industrial as IES, enquanto organismos se utilizam de uma grande quantidade dos recursos disponíveis, isso se deve, principalmente, ao grande fluxo de pessoas, informações e atividades desenvolvidas. É possível afirmar a existência de inputs e outputs, conforme apresentado na Figura 1. 
Figura 1 Principais fluxos de um campus universitário

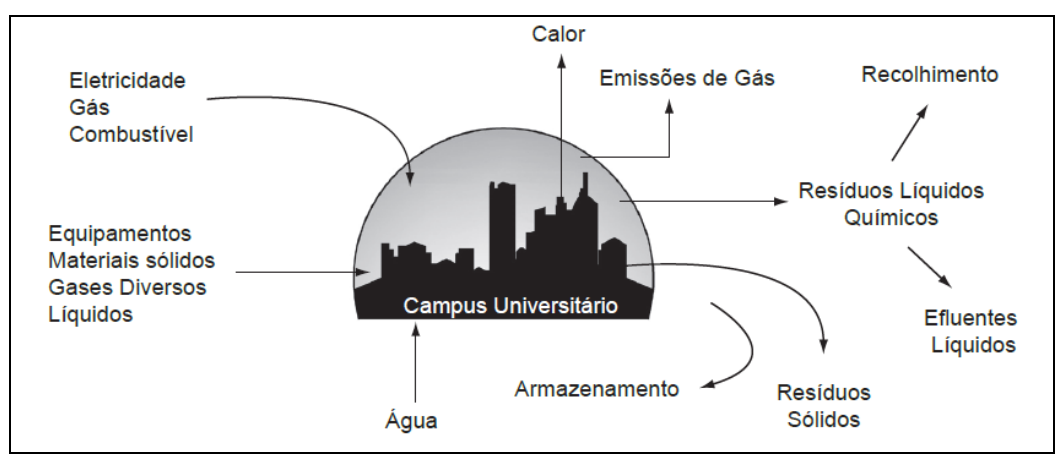

Fonte: Careto e Vendeirinho (2003, p. 9)

Desta maneira, verifica-se que as instituições possuem um grande passivo ambiental, tendo a necessidade de incorporarem o desenvolvimento sustentável na consecução de suas atividades. As universidades devem pesquisar e desenvolver práticas sustentáveis em seus espaços institucionais, onde deve prevalecer a eliminação de desperdícios e a redução do consumo de recursos naturais, implicando necessariamente em uma mudança de comportamentos (TAUCHEN; BRANDLI, 2006). A implantação de sistemas de gestão ambiental nos campi universitários serve como modelos e exemplos práticos de gestão sustentável para a sociedade.

Reconhece-se que as IES estão em constante intervenção com a sociedade. De acordo com os estudos de Fouto (2002), as IES apresentam quatro níveis de intervenção com a sociedade, sendo: (1) educação dos tomadores de decisão para um futuro sustentável; (2) investigação de soluções, paradigmas e valores que sir $\neg$ vam uma sociedade sustentável; (3) operação dos campi universitários como modelos e exemplos práticos de sustentabilidade à escala local; e (4) coordenação e comunicação entre os níveis anteriores e entre estes e a sociedade. Estes níveis de intervenção e sua inter-relação podem ser observados na Figura 2: 
Figura 2 O papel da universidade na sociedade, relativo ao desenvolvimento sustentável

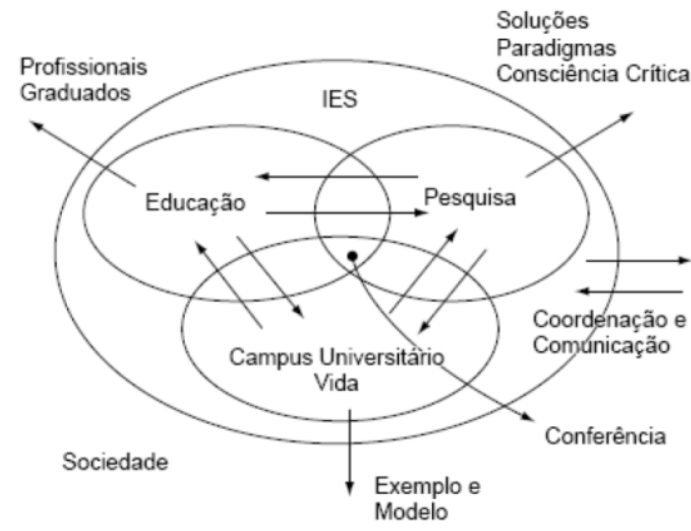

Fonte: Fouto (2002)

Para Weenen (2000), há muitas formas de as universidades promoverem o desenvolvimento sustentável, sejam elas nas ações de planejamento, gestão, desenvolvimento, ensino, pesquisa, operações, extensão, compras, transporte, construções, entre outros. Alshuwaikh e Abubakar (2008) argumentam que um campus sustentável deve ser ambientalmente saudável, com uma próspera economia por meio de racionalização no consumo de energia e conservação de recursos, redução de resíduos e com uma gestão ambiental eficiente; devendo promover a equidade e justiça social e exportar estes valores para o comunidade. Segundo Evangelinos et al. (2009) a promoção da sustentabilidade no contexto das instituições de ensino superior pode ser conseguida através de ensino e pesquisa (DELAKOWITZ; HOFFMAN, 2000), a melhoria da gestão ambiental (BONNET et al., 2002) e transmissão de conhecimento para a sociedade (OWENS; HALFACREHITCHCOCK, 2006).

Assim, as IES são vistas como agentes especialmente equipados para liderar o caminho em busca do desenvolvimento sustentável (TAUCHEN; BRANDLI, 2006), uma vez que se constituem em espaços para "reflexão, formação e difusão de novas concepções de desenvolvimento e sustentabilidade, participando numa perspectiva mais ampla do estabelecimento de sociedades mais justas, solidárias e ambientalmente sustentáveis" (BRASIL, 2007, p. 25).

A respeito de práticas sustentáveis Simkins e Nolan (2004) ressaltam a importância de assegurar que as pessoas que trabalharão na melhoria do desempenho ambiental da instituição estejam preparadas, bem como que haja uma organização interna, onde cada membro seja responsável por uma função, uma área. Contudo, na visão de Brandli et al. (2010), alguns 
aspectos dificultam a inserção de práticas sustentáveis nas IES, sendo: o sistema burocrático e hierárquico rígido; a falta de conscientização e comprometimento dos agentes envolvidos (docentes, discentes e administração); e a inexistência de definição de políticas institucionais de curto e longo prazo.

Mesmo assim, o encontro de novos caminhos para a conscientização de práticas mais sustentáveis passou a ser um discurso presente em várias IES na última década (RYAN et al., 2010), e merece atenção da academia em sua análise e efetividade.

\section{MÉTODO DE INVESTIGAÇÃO}

Nesta seção é apresentado o método de estudo aplicado, a justificativa para escolha do método e os procedimentos para coleta e análise dos dados.

\subsection{MÉTODO DE PESQUISA}

O método de pesquisa utilizado foi o de estudo de caso único, considerado adequado quando o foco do estudo está em fenômenos contemporâneos nos quais há pouco controle sobre os acontecimentos, adotando uma abordagem holística (YIN, 2010).

Para a seleção do estudo de caso, obedeceu-se os critérios de escolha estabelecidos por Yin (2010), sendo que neste estudo, utilizou-se o desenvolvimento teórico para ajudar a selecionar o caso, desenvolver o protocolo de coleta de dados e organizar as estratégias iniciais de coleta e análise de dados.

\subsection{ESCOLHA DA UNIDADE DE ANÁLISE}

Yin (2010) distingue quatro tipos de projetos de estudo de caso, sendo este considerado como estudo de caso único (holístico) e descritivo. A unidade de análise escolhida foi a Universidade Federal de Santa Maria (UFSM).

No que se refere à justificativa para o projeto de estudo de caso único, dentre as diversas definições de Yin (2010), este refere-se a um caso representativo ou típico. Para o autor, o objetivo neste caso é captar as circunstâncias e as condições de uma situação diária ou de um lugar comum. As lições aprendidas desses casos são presumidamente informativas sobre experiências médias das instituições.

De acordo com seu Plano de Desenvolvimento Institucional - PDI a Universidade tem como missão principal "construir e difundir conhecimento, comprometida com a formação de 
pessoas capazes de inovar e contribuir com o desenvolvimento da sociedade, de modo sustentável" (UFSM, 2011). Os eixos norteadores, definidos pelo PDI, enquanto elo entre as ações das diversas áreas de atuação da UFSM e a sua filosofia são: foco na inovação e na sustentabilidade (grifo nosso); inclusão, acesso e acessibilidade, cooperação e inserção social; qualificação das atividades acadêmicas; valorização das pessoas; expansão acadêmica qualificada da UFSM; e, otimização da gestão institucional (UFSM, 2011).

\subsection{INSTRUMENTO E COLETA DE DADOS}

Uma das principais características do estudo de caso volta-se a utilização de várias fontes de evidências (YIN, 2010). Nesse sentido, este envolveu levantamentos por meio de documentos da IES, arquivos e aplicação de questionário junto aos servidores da UFSM. Para o autor, as várias fontes são altamente complementares e o ideal seria o uso de tantas fontes quanto possível em um estudo de caso.

A coleta de dados junto aos servidores da UFSM foi operacionalizada por meio de um levantamento do tipo survey, de modo a verificar o conhecimento que os servidores da Universidade possuíam sobre temas importantes para o desenvolvimento sustentável da IES e da Administração Pública em geral.

O instrumento de coleta de dados foi desenvolvido a partir da literatura revisada. Nele solicitou-se aos respondentes que informassem em uma escala de 1 (nenhum conhecimento) a 5 (grande conhecimento), seu grau conhecimento sobre a temática. As variáveis englobavam os seguintes temas: o Plano de Gestão Logística Sustentável, a Agenda da Administração Pública, a Agenda 21, a Política Nacional de Resíduos Sólidos e a Logística Reversa.

Em seguida, foi solicitado que o respondente informasse quais atividades ligadas ao desenvolvimento sustentável eram desenvolvidas em seu setor e que poderiam ser difundidas às demais pessoas. Estas questões objetivaram verificar o comprometimento dos servidores com as práticas sustentáveis e se essas práticas são recorrentes nos diversos setores da instituição.

O questionário foi elaborado na ferramenta Google Docs e enviado por meio de e-mail a todos os servidores da Universidade (num total de 4.690 servidores em 16-06-2014, sendo que 1.880 eram docentes e outros 2.810 técnico-administrativos). $\mathrm{O}$ acesso ao questionário foi permitido por um período de dois meses (entre 15/04/2014 e 15/06/2014). A amostra da pesquisa foi constituída a partir do número de servidores que retornaram o questionário, 
representando assim 649 servidores (236 são docentes e 413 técnicos-administrativos). Essa amostra resultante não pode ser considerada probabilística, observando que a probabilidade de inclusão de cada membro da população na amostra não é conhecida e nem equivalente (MALHOTRA, 2006).

\subsection{TRATAMENTO E ANÁLISE DOS DADOS}

A apreciação dos dados ocorreu por meio de tabulação dos dados quantitativos e posterior análise univariada, por meio da ferramenta SSPS Statistics. Salienta-se que o respondente era impossibilitado de enviar o questionário, caso alguma questão não estivesse preenchida. Dessa forma, a pesquisa não apresentou nenhum dado faltante. Os outliers univariados foram verificados por meio do teste $\mathrm{Z}$ score, não sendo encontrados valores atípicos na amostra. Como medida complementar na preparação da matriz de dados, procedeu-se a verificação da normalidade dos dados, por meio do teste de KolmogorovSmirnov. Não foram verificadas variáveis anormais.

Já a análise dos dados qualitativos foi feita por meio de análise de conteúdo. Para tal, inicialmente os resultados foram transcritos e agrupados por variáveis de análise. Em seguida, foram analisados, tomando por base a revisão da literatura.

\section{RESULTADOS}

Esta seção apresenta os resultados auferidos por meio da survey e dos levantamentos documentais. Os resultados apresentados buscam descrever o conhecimento em relação às temáticas sustentáveis dentro da UFSM e ainda verificar atividades que são desenvolvidas nas unidades universitárias. Assim, busca-se abordar os impactos que estas situações causam no desenvolvimento sustentável da UFSM.

\subsection{GRAU DE CONHECIMENTO DOS SERVIDORES DA UFSM SOBRE AS TEMÁTICAS SUSTENTÁVEIS}

$\mathrm{Na}$ Tabela 1 são apresentados os resultados referentes ao grau de conhecimento apresentado pelos servidores da UFSM sobre as temáticas sustentáveis características de organizações públicas, que possuem enfoque no desenvolvimento sustentável. 
Tabela 1 Medidas de tendência central e dispersão das variáveis de conhecimento sobre temáticas sustentáveis

\begin{tabular}{lcccc}
\hline & Média & Mediana & Modo & Desvio Padrão \\
\hline Plano de Gestão de Logística Sustentável da UFSM & 1,77 & 1,00 & 1 & 1,041 \\
Agenda Ambiental da Administração Pública (A3P) & 1,59 & 1,00 & 1 & 0,887 \\
Agenda 21 & 2,01 & 2,00 & 1 & 1,145 \\
Política Nacional de Resíduos Sólidos & 2,22 & 2,00 & 1 & 1,162 \\
Logística Reversa & 1,93 & 1,00 & 1 & 1,173 \\
\hline
\end{tabular}

Fonte: elaborado pelos autores (2015)

A maior média apresentada é com relação à Política Nacional de Resíduos Sólidos (média 2,22), representando um conhecimento entre "pouco conhecimento" e "razoável conhecimento". De outra parte, a menor média é relacionada à Agenda Ambiental da Administração Pública (A3P) (média 1,59), o que representa um conhecimento entre "nenhum conhecimento" e "pouco conhecimento". O Plano de Gestão de Logística Sustentável da UFSM é o tópico que apresentou a segunda menor média de conhecimento entre os respondentes $(1,77)$.

Os tópicos Agenda 21 e Política Nacional de Resíduos Sólidos apresentaram, por meio da mediana observada, o grau de conhecimento "pouco conhecimento", como ponto de tendência central da amostra pesquisa. Já os tópicos Plano de Gestão de Logística Sustentável, A3P e Logística reversa, apresentaram como ponto de tendência central o grau de conhecimento "nenhum conhecimento". As temáticas Agenda 21 e Política Nacional de Resíduos Sólidos foram as que apresentaram maior familiaridade para os servidores da UFSM, elas apresentaram uma média de 2,01 e 2,22, respectivamente.

Por meio da análise da moda, é possível observar que o grau de conhecimento "nenhum conhecimento" foi o item mais frequentemente assinalado pelos respondentes em todos os tópicos pesquisados. Já o desvio-padrão apresentado demonstra uma relativa proximidade das respostas em relação à média apresentada pelos tópicos.

Os resultados demonstram que os servidores da UFSM possuem um baixo conhecimento sobre os tópicos apresentados. Em linhas gerais, o grau de conhecimento demonstrado é insuficiente para que se considerem os servidores da UFSM conhecedores das temáticas apresentadas. Fato que pode acarretar em um ineficaz desenvolvimento sustentável dentro da UFSM. As temáticas propostas aos servidores na pesquisa são comumente utilizadas pela Administração Pública na busca por um ambiente pautado no desenvolvimento sustentável, pois se tratam das principais iniciativas institucionais que atualmente são 
encontradas na gestão pública. Percebe-se assim, a necessidade de maior disseminação referente ao conhecimento dos tópicos apresentados.

Os resultados de nosso estudo corroboram com os dados apresentados por Silva (2014) que demonstrou que um índice de 95,65\% dos entrevistados não entendiam claramente o que era desenvolvimento sustentável, ainda, segundo o autor, grande parte dos entrevistados não creem que tenham qualquer responsabilidade em possíveis mudanças de atitudes. Já nos estudos apontados por Nascimento, Virgínio e Lopes (2015) 60\% dos funcionários apresentavam dificuldades para explicar o conceito de sustentabilidade e $96 \%$ desconheciam a intenção de implantação de um Programa Socioambiental na instituição pesquisada e, alguns funcionários, ainda apresentavam determinada dificuldade para associar o comportamento sustentável com a qualidade de vida no ambiente de trabalho.

Em estudo desenvolvido por Yuan, Zuo e Huisingh (2013) foi descoberto que professores e funcionários na China têm relativamente baixa consciência ambiental e percebem o seu papel como não tão importante. Já estudos desenvolvidos em universidades da Malásia mostram que funcionários universitários têm conhecimento limitado do desenvolvimento sustentável, no entanto apresentam uma atitude fortemente positiva em relação sustentabilidade e entendem que possuem papel importante na educação para o desenvolvimento sustentável (DERAHIM et al., 2012).

Outra barreira existente para o desenvolvimento de um conhecimento sustentável é a diferença nas percepções sobre sustentabilidade e a falta de uma única definição geral de desenvolvimento sustentável. Além disso, a imagem de desenvolvimento sustentável varia em diferentes partes da universidade e entre os diferentes indivíduos. Apesar dos esforços contínuos nas políticas de desenvolvimento sustentável nas universidades, a sua institucionalização leva um longo tempo para desenvolver (SAMMALISTO; SUNDSTRÖM; HOLM, 2015).

Ainda que não exista uma percepção consistente por parte dos servidores, a necessidade de inserção destes tópicos no ambiente organizacional é primordial para o sucesso de uma política de desenvolvimento sustentável. Um estudo sueco demonstrou que treinamento e comunicação dentro de uma implementação do sistema de gestão ambiental aumentou tanto a consciência para questões ambientais quanto a compreensão dos professores e funcionários de seu papel no trabalho ambiental (SAMMALISTO; BRORSON, 2008). Por meio da mudança nos valores e práticas, os servidores públicos se transformarão em novos 
atores e contribuirão para a construção de uma nova cultura organizacional pública, proporcionando um meio ambiente mais equilibrado, com a inserção de critérios sociais e ambientais na rotina administrativa, acarretando em um diferencial estratégico (SOUSA; CARVALHO, 2015).

A A3P Agenda Ambiental da Administração Pública (A3P) propõe, por meio da capacitação, uma série de ações que contribuem para o desenvolvimento de competências institucionais e individuais, fornecendo aos servidores oportunidade para desenvolver habilidades e atitudes para um melhor desempenho das suas atividades (MMA, 2009). Teoricamente, as IES se encontram em posição privilegiada para melhorar o seu comportamento ambiental, pois, se por um lado são geradoras de problemas ambientais, por outro lado dispõem do conhecimento e competências necessárias para abordar esses mesmos problemas (GRAEDEL, 2002).

\subsection{PRÁTICAS SUSTENTÁVEIS ADOTADAS PELOS SERVIDORES DA UFSM EM SEUS AMBIENTES DE TRABALHO}

A A3P tem como principal objetivo estimular a reflexão e a mudança de atitude dos servidores para que os mesmos incorporem os critérios de gestão socioambiental em suas atividades rotineiras (MMA, 2009, p. 33).

As atividades relacionadas na cartilha da A3P são agrupadas em eixos temáticos, os quais são: uso racional dos recursos naturais e bens públicos, Gestão adequada dos resíduos gerados, qualidade de vida no ambiente de trabalho, sensibilização e capacitação dos servidores e licitações sustentáveis (Figura 3).

Figura 3 Eixos temáticos da A3P

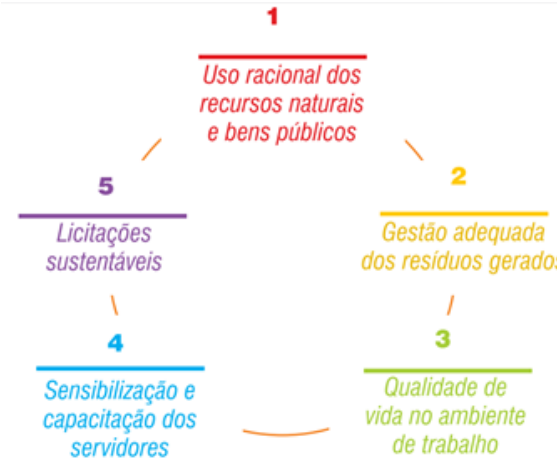

Fonte: Ministério do Meio Ambiente (MMA), 2009. 
A cartilha da A3P aponta algumas atividades que podem ser realizadas para o uso racional dos recursos naturais e bens públicos. Referente à economia de papel enumera ações, tais como: a utilização de e-mail para comunicação interna e externa; quando for imprimir confira sempre no monitor se não há nenhum erro; utilizar a frente e o verso das folhas, sempre que possível.

Para economia de energia: Dê preferência à iluminação natural, abrindo janelas, cortinas e persianas; Ao sair para o almoço, desligue, ao menos, o monitor do computador; Não deixe computadores e outros equipamentos elétricos ligados por muito tempo sem uso.

Para economia de água: Coloque ou sugira a colocação de adesivos com mensagens educativas lembrando a todos da necessidade do bom uso da água no ambiente de trabalho; Substitua as torneiras e as caixas de descargas por outras mais econômicas.

Outras atividades com relação à economia de energia e de água podem ser realizadas na instituição e dependem de uma adequação dos prédios da instituição. Ao buscar uma construção sustentável é possível instalar sistemas de captação de chuva, maior eficiência energética privilegiando a ventilação e a luminosidade natural.

Infere-se que a gestão adequada dos resíduos gerados busca, em um primeiro momento, o combate ao desperdício e, posteriormente, a destinação correta dos resíduos. Tais atividades estão diretamente ligadas à política dos 5R's proposta na $\mathrm{A} 3 \mathrm{P}$ : repensar, reduzir, reutilizar, reciclar e recusar. Nesta temática estão ligadas as ações de redução na geração de resíduos e a destinação correta dos resíduos gerados, implantação da coleta de seletiva de materiais o que proporcionaria um aumento no número de materiais reciclados. As instituições governamentais devem buscar a mudança de hábitos e atitudes internas, promovendo uma nova cultura institucional de combate ao desperdício (MMA, 2009, p. 28).

A qualidade de vida no ambiente de trabalho dos servidores busca facilitar e satisfazer as necessidades do trabalhador ao desenvolver suas atividades na organização. Nesta etapa se evidenciam as ações que proporcionem melhorias no ambiente de trabalho, promoção da saúde e segurança e desenvolvimento das capacidades humanas. Diversas ações podem ser realizadas nesse sentido, tais como: aproveitamento das habilidades e autonomia na atividade desenvolvida; criação de áreas comuns para integração de servidores; promoção dos relacionamentos interpessoais; privacidade pessoal; ginástica laboral e outras atividades; salubridade dos ambientes. 
No que tange à sensibilização e à capacitação dos servidores busca-se desenvolver competências institucionais e individuais fornecendo oportunidades para melhoria no desempenho de suas atividades. O desenvolvimento de um plano de capacitação, a realização de palestras, cursos, fóruns, apresentações teatrais, são ações que buscam estimular uma sensibilização e capacitação dos servidores. Esta temática é de extrema importância para ampliação do conhecimento dos servidores no que se refere aos tópicos de desenvolvimento sustentável apresentados.

A temática relacionada às licitações sustentáveis foca na inserção do aspecto sustentável nas compras realizadas pela Administração Pública. Para promoção do desenvolvimento sustentável é necessário que o agente tomador de decisão ao definir uma compra deve fundamentar sua decisão não só no aspecto econômico, mas também no aspecto ambiental e social. Melhorias nas especificações de materiais sustentáveis, disponibilização de materiais sustentáveis para utilização e melhoria na contratação de serviços são ações que auxiliam no desenvolvimento sustentável da instituição.

Os resultados da pesquisa mostram que por desconhecimento destas ações ou por desinteresse dos servidores da Universidade em análise, essas ações não são realizadas por todos os servidores (grifo nosso). Na pesquisa realizada, 212 respondentes (32,66\% do total) informaram que não é realizada nenhuma atividade sustentável ou que não possuem conhecimento sobre qualquer tipo de atividade que seja realizada na sua unidade de trabalho. A ausência de ações nesse sentido pode ser explicada, em partes, pela dificuldade apontada de Wright e Wilton (2012) de inserir a sustentabilidade em todos os níveis de uma universidade.

No que se refere à economia de papel, 138 respondentes $(21,26 \%$ do total) afirmaram que desenvolvem algum tipo de atividade que tem como foco a racionalização na utilização de papel. Foram identificadas várias atividades, tais como: impressão frente e verso, envio de documentos em formato digital, reaproveitamento de folhas para rascunho, conferência do documento antes de imprimir. Em pesquisa realizada por Silva (2014) 70\% dos entrevistados afirmaram que possuem o hábito de reutilizar materiais e fazer rascunhos de papéis inutilizados, no entanto, somente $17,4 \%$ se interessaram em participar de qualquer tipo de oficina envolvendo papéis e/ou materiais recicláveis, outro dado importante da pesquisa apresentada pelo autor é que $89 \%$ dos entrevistados acreditam que a utilização de novas tecnologias, como a utilização de arquivos digitais, poderia ser uma das soluções para a redução na utilização de papel. 
A troca de copos descartáveis por copos de vidros ou outros materiais de maior durabilidade foi apontada por 97 respondentes $(14,95 \%$ do total) como uma das práticas adotadas para redução na geração de resíduos, nos estudos apontados por Nascimento, Virgínio e Lopes (2015) o índice de entrevistados que manifestaram utilizar canecas próprias para o consumo do café distribuído durante a jornada de trabalho foi de $40 \%$. A adoção de práticas para redução do consumo de água foi apontada por 30 respondentes (4,62\% do total), que engloba a instalação de torneiras automáticas nos banheiros e aproveitamento de água da chuva.

No que tange à redução no consumo de energia elétrica, somente 19 entrevistados (2,92\% do total) afirmaram que desenvolvem algum tipo de atividade. Algumas das práticas elencadas para redução do consumo de energia elétrica referem-se a privilegiar a utilização de luz solar e a ventilação natural e a substituição de lâmpadas.

Ressaltando a importância na economia de recursos e nas atividades de racionalização de materiais desenvolvidas pelos servidores, Jain e Pant (2010) apontaram em seu estudo de caso que as principais preocupações na gestão ambiental nos sistemas universitários foram identificados como o consumo de energia, gestão de resíduos, prevenção da poluição e conservação de recursos.

Não obstante, durante o desenvolvimento da pesquisa, alguns respondentes enumeraram atividades mais complexas, frutos de projetos de pesquisa e extensão, do que aquelas desenvolvidas habitualmente nos setores de trabalho. Verificou-se assim, que a UFSM possui diversos cursos e projetos ligados às questões ambientais e sociais. Tal fato reforça a existência de uma grande diversidade de conhecimento e de competências dentro da instituição.

Como atividades desenvolvidas, enumeram-se projetos de aproveitamento de resíduos para utilização em camas aviárias e a elaboração de biocombustível. O aproveitamento da água da chuva, produção de biodiesel com óleo de fritura, utilização de energias renováveis solar térmica, solar fotovoltaica, eólica, sistemas híbridos e biomassa, atividades de irrigação por gotejamento, utilização de placas solares para bombeamento de água e energia, compostagem, uso de cobertura morta para reduzir o consumo de água, utilização de telhas transparentes para iluminação, distribuição de plantas para escolas, entre outras.

Dentre as atividades de ensino, foram citados projetos e pesquisas de extensão sobre diversos assuntos ligados ao desenvolvimento sustentável, tais como: pesquisa referente ao 
comportamento de alunos/docentes no descarte de resíduos sólidos, experimentos com vistas à educação sustentável no ensino médio, pesquisas em geração de energia a partir de fontes de energia renováveis, criação de casas populares sustentáveis, estudo da sustentabilidade sob a temática multidimensional (ambiental, social, cultural, técnica, ética) como tema transversal.

Contudo, essas ações desenvolvidas referem-se a esforços individuais na busca de mudar hábitos, comportamentos e padrões de consumo, mas, infelizmente, ainda são pouco representativas dentro de um universo grande de servidores. Para que essas mudanças sejam possíveis é necessário o engajamento individual e coletivo, pois apenas dessa forma será possível a criação de uma nova cultura institucional de sustentabilidade das atividades do setor público, sejam essas relacionadas à área meio ou à área finalística (MMA, 2009, p. 45).

Embora professores e servidores das universidades ainda veem a sustentabilidade como periférica às suas funções (WALS, 2014) e estão nas fases iniciais do processo de aprendizagem (MULDER; SEGALAS; FERRER-BALAS, 2012), eles são os agentes de mudança que podem e irão se empenhar na educação para o desenvolvimento sustentável. (BARTH; RIECKMANN, 2012).

\section{CONCLUSÃO}

Tomando por base o papel das IES na formação de futuros profissionais e tomadores de decisão e ainda no fornecimento de informações e conhecimentos, estas, teoricamente, devem incorporar os princípios e práticas da sustentabilidade, seja para iniciar um pro $\neg$ cesso de conscientização em todos os seus níveis - atin $\neg$ gindo professores, funcionários e alunos ou para tomar decisões fundamentais sobre planejamento, treinamento, operações ou atividades comuns em suas áreas físicas (TAUCHEN; BRANDLI, 2006).

A partir dos resultados auferidos neste estudo, verificou-se que há ampla deficiência no conhecimento referente a temáticas sustentáveis da Administração Pública por parte dos servidores da UFSM. Ainda, a ampla maioria dos respondentes não desenvolvem atividades rotineiras visando a racionalização dos recursos. É possível afirmar que o desenvolvimento sustentável ainda é algo pouco palpável na Universidade e que depende de maior engajamento dos atores envolvidos nesse processo.

A busca pelo desenvolvimento sustentável deve ser uma política de âmbito institucional, mas desenvolvida em nível operacional. Esta pesquisa revelou que apesar da 
sustentabilidade ser um tópico presente nos planos de desenvolvimento da instituição, não existe de fato uma política institucional voltada para o desenvolvimento sustentável.

Por fim, o presente artigo atinge seu objetivo na medida em que estimula a reflexão sobre as ações desenvolvidas no ambiente organizacional das IES e proporciona o desenvolvimento de novos estudos no que concerne ao relacionamento entre IES e o desenvolvimento sustentável, para que incorpore na prática os valores disseminados por ela como instituição formadora de conhecimento, contudo,

Torna-se cogente destacar algumas limitações deste trabalho, pois ele reflete os resultados de um estudo de caso único e holístico. Embora o estudo de caso possa ser considerado típico de universidades públicas brasileiras, os resultados ora apresentados não devem ser tomados como certos e generalizáveis, sem a ampliação da investigação sobre o tema em outras IES. Não obstante, a aplicação de outros métodos de coleta e análise de dados como entrevistas em profundidade servem como parâmetros futuros de investigação.

\section{REFERÊNCIAS}

ALSHUWAIKHAT, H. M.; ABUBAKAR, I. An integrated approach to achieving campus sustainability: assessment of the current campus environmental management practices. Journal of Cleaner Production, v. 16, p. 1777-1785, nov. 2008.

AZEVEDO, R. A. B. A sustentabilidade da agricultura e os conceitos de sustentabilidade estrutural econjuntural. Revista Agricultura Tropical, Cuiabá, v.6, n.1, p. 9-42, 2002.

BARTH, M., RIECKMANN, M. Academic staff development as a catalyst for curriculum change towards education for sustainable development: an output perspective. Journal of Cleaner Production, v. 26, p. 28-36, mai. 2012.

BONNET, J. F. et al. Analysis of electricity and water end-uses in university campuses: case-study of the University of Bordeaux in the framework of the Ecocampus European Collaboration. Journal of Cleaner Production, v. 10 (1), p. 13-24, fev. 2002.

BRANDLI, L. B. et al. The Latin America Meeting of Sustainable Universities (I ELAUS): results and possibilities. In: ERSCP-EMSU CONFERENCE, Delft, The Netherlands, 2010.

BRASIL. Mapeamento da Educação Ambiental em Instituições Brasileiras de Educação Superior: elementos para políticas públicas. Série Documentos Técnicos, $\mathrm{n}^{\circ} 12$. Brasília: MMA/ME, 2007.

CARETO, H.; VENDEIRINHO, R. Sistemas de Gestão Ambiental em Universidades: Caso do Instituto Superior Técnico de Portugal. Relatório Final de Curso, 2003. 
Disponível em: <http://meteo.ist.utl.pt/ jjdd/LEAMB/LEAmb\%20TFC\%20site\%20v1/20022003/HCareto_RVendeirinho\%20artigo.pdf> Acesso em: 07 de jul. 2014.

CAVAlCANTI, M. Administração Pública e Agenda Ambiental - A3P - Considerações sobre a implementação nos órgãos públicos. Revista Controle, v. 10, n. 1, p. 196-216, janjul 2012.

CIEGIS, R.; RAMANAUSKIENE, J.; MARTINKUS, B. The concept of sustainable development and its use for sustainability scenarios. [S. 1.]: Inzinerine EkonomikaEngineering Economics, 2009.

CMMAD, Comissão Mundial sobre Meio Ambiente e Desenvolvimento. Nosso Futuro Comum. 2 ed. Rio de Janeiro, Ed. Fundação Getúlio Vargas, 1991.

CONSALTER, M. A. S. Sistema de produção lavoura-pecuária: uma abordagem para a construção de indicadores integrados de sustentabilidade. 2008. Tese (Doutorado em Agronomia) - Universidade Federal do Paraná, Curitiba, 2008.

COUTO, H. L. G. do; COELHO, C. Fatores críticos no comportamento do gestor público responsável por compras sustentáveis: diferenças entre consumo individual e organizacional. Revista de Administração Pública, Rio de Janeiro, v. 49, n. 2, p. 519-543, abr. 2015.

DELAKOWITZ, B.; HOFFMANN, A. The Hochschule Zittau/G€orlitz: Germany's first registered environmental management (EMAS) at an institution of higher education. International Journal of Sustainability in Higher Education, v. 1, n. 1, p. 35-47, 2000.

DEPONTI, C. M.; ALMEIDA, J. Indicadores para a avaliação da sustentabilidade em contextos de desenvolvimento rural local. In: Congresso Latino-Americano de Sociologia Rural, 2002, Porto Alegre, Anais, p.1-21, 2002.

DERAHIM, N. et al. UKM's staff perspective on sustainability and its contribution towards a sustainable university. Procedia - Social and Behavorial Sciences. v. 59, p. 376381 , out. 2012.

DOBES, V. EMS and change of guiding ideas in direction of sustainability. In: Paper Presented at the 7th European Roundtable on Cleaner Production, Lund, Sweden, 2001.

ELKINGTON, J. Cannibals with forks: the triple bottom line of 21 st century business. Oxford: Capstone, 1999. 402p.

EVANGELINOS, K. I.; JONES, N.; PANORIOU, E. M. Challenges and opportunities for sustainability in regional universities: a case study in Mytilene, Greece. Journal of Cleaner Production, v. 17, n. 12, p. 1154-1161, ago. 2009.

FREITAS, C. L. Avaliação de Sustentabilidade em Instituições Públicas Federais de Ensino Superior (IFES): proposição de um modelo baseado em sistemas gerenciais de 
avaliação e evidenciação socioambiental. Dissertação (Mestrado em Contabilidade), Universidade Federal de Santa Catarina, Florianópolis, 2013.

FOUTO, A. R. F. O papel das universidades rumo ao desenvolvimento sustentável: das relações internacionais às práticas locais. Dissertação (Mestrado em Gestão e Políticas Ambientais Relações Internacionais do Ambiente), Universidade Nova de Lisboa, 2002.

GALLI, A. Educação ambiental como instrumento para o desenvolvimento sustentável. Paraná: Juruá. 2008.

GRAEDEL, T. E. Quantitative sustainability in a college or university setting. International Journal of Sustainability in Higher Education. v. 3, n. 4, p. 346-58, 2002.

JACOBI, P. Educação ambiental, cidadania e sustentabilidade. Cadernos de Pesquisa, São Paulo, n. 118, p. 180-203, 2003.

JAIN, S.; PANT, P. Environmental management systems for educational institutions. A case study of TERI University, New Delhi, International Journal of Sustanability in Higher Education, v. 11, n. 3, p. 236-249, 2010.

LEAL FILHO, W.; MANOLAS, E.; PACE, P. The future we want: key issues on sustainable development in higher education after Rio and the $U N$ decade of education for sustainable development, International Journal of Sustanability in Higher Education, v. 16, n. 1, p. 112-129, 2015.

MINISTÉRIO DO MEIO AMBIENTE - MMA. Agenda Ambiental na Administração Pública. Brasília, 2006.

MULDER, K. F.; SEGALAS, J.; FERRER-BALAS, D. How to educate engineers for/in sustainable development Ten years of discussion, remaining challenges. International Journal of Sustanability in Higher Education, v. 13, n. 3, p. 211-218, 2012.

NASCIMENTO, M. de M.; VIRGÍNIO, M. V. de O.; LOPES, L. R. Educação Ambiental na Administração Pública: A Implantação da A3P na Universidade Federal do Vale do São Francisco/Univasf-PE. Revista Eletrônica em Gestão, Educação e Tecnologia Ambiental, Santa Maria, v. 19, n. 2, p. 493-501, mai./ago. 2015.

OWENS, K. A.; HALFACRE-HITCHCOCK, A. As green as we think? The case of the College of Charleston green building initiative. International Journal of Sustanability in Higher Education, v. 7, n. 2, p. 114-128, 2006.

QUADROS, D. S. Subsídios para o Sistema de gestão ambiental da Universidade Regional de Blumenau. (Dissertação) Mestrado em Administração Gestão Moderna de Negócios. Centro de Ciências Sociais e Aplicadas, Universidade Regional de Blumenau. Blumenau, 1999. 
RYAN, A. et al. Sustainability in higher education in the Asia-Pacific: developments, challenges, and prospects. International Journal of Sustainability in Higher Education. v. 11, n. 2, p. 106-119, 2010.

SACHS, I. Estratégias de transição para o século XXI: desenvolvimento e meio Ambiente. São Paulo: Studio Nobel e Fundação de Desenvolvimento Administrativo (FUNDAP), 1993.

SAMMALISTO, K.; BRORSON, T. Training and communication in the implementation of environmental management systems (ISO 14001): a case study at the University of G€avle, Sweden, Journal of Cleaner Production, v. 16, n. 3, p. 299-309, 2008.

SANTOS, T. Organizações da sociedade civil e as construções teóricas contemporâneas acerca da sustentabilidade. In: VI Conferência Regional de ISTR Para América Latina Y El Caribe, Salvador/BA, Anais... 2007.

SILVA, V. R. da. Práticas Sustentáveis: o uso Consciente do Papel e o Reaproveitamento de Materiais. Revista Laborativa. v. 3, (Supl. 1), p. 79-89, dez. 2014.

SIMKINS, G.; NOLAN, A. Environmental Management Systems in Universities. (Occasional Paper for the Environmental Association for Universities and Colleges). EAUC. Disponível em: < http://www.eauc.org.uk/sorted/files/ems_eauc_paper_1.pdf $>$ Acesso em: 11 de jul. 2014.

SOUSA, E. da S.; CARVALHO, D. B. de. Educação para Gestão Ambiental: Um Estudo dos Instrumentos Propostos pela Administração Pública Federal voltados ao consumo sustentável. Revista Brasileira de Educação Ambiental, São Paulo, v. 10, n. 2, p. 283-300, 2015 .

TAUCHEN, J.; BRANDLI, L. L. A gestão ambiental em instituições de ensino superior: Modelo de implantação em Campus Universitário. Gestão e Produção, v.13, n.3, p. 503 515, set/dez. 2006.

UNIVERSIDADE FEDERAL DE SANTA MARIA. Plano de Desenvolvimento Institucional 2011-2015. Disponível em:

$<$ http://w3.ufsm.br/proplan/images/stories/file/COPLIN/PDI-2011-2015.pdf $>$ Acesso em: 01 jul. 2014.

YIN, R. K. Estudo de caso: planejamento e métodos. 4. Ed. Porto Alegre: Bookman, 2010.

YUAN, X.; ZUO, J.; HUISINGH, D. Green universities in China e what matters? Journal of Cleaner Production, n. 61, p. 36-45, 2013.

WALS, A. E. J. Sustainability in higher education in the context of the UN DESD: a review of learning and institutionalization processes. Journal of Cleaner Production, v. 62, p. 8-15, 2014. 
WEENEN, H. Towards a vision of a sustainable university. International Journal of Sustainability in Higher Education, v. 1, n. 1, p. 20-34, 2000.

WRIGHT, T.; WILTON, H. Facilities management directors' conceptualizations of sustainability in higher education. Journal of Cleaner Production, v. 31, p. 118-125, 2012. 